\title{
Gloss measurement in detection of surface quality of pharmaceutical tablets: a case study of screening of genuine and counterfeit antimalaria tablets
}

\author{
Prince Bawuah ${ }^{*} \mathbb{D}$, Pertti Pääkkönen and Kai-Erik Peiponen
}

\begin{abstract}
Background: The surface quality of pharmaceutical tablets plays an important role, especially in the drug release of a tablet and hence affects the bioavailability of the drug. Furthermore, important tablet's properties such as coating uniformity and mechanical integrity that can be predicted based on the known surface properties manifest the inevitable link between a tablet's surface property (e.g. Gloss, average surface roughness) and its efficacy.

Methods: In this article, laboratory based and handheld glossmeters as well as an optical profilometer were utilized for the detection of the surface properties of commercially available pharmaceutical tablets.

Results: As a case study, we measured the surface properties of authentic and counterfeit antimalarial tablets. Clear differences between authentic and fake tablets were detected based on the measured average surface roughness and gloss values of the tablet samples.

Conclusions: It is worth mentioning that this is the first time we have utilized both glossmeters for the purpose of surface quality investigation of real pharmaceutical tablets. It is therefore suggested that the handheld glossmeter is a useful device for quick inspection of tablet's surface quality both in the production environments as well as for field screening of fake tablets.
\end{abstract}

Keywords: Gloss, Surface roughness, Quality screening, Antimalaria tablets, Artemether, Lumefantrine

\section{Background}

The ability to critically assess the surface properties of a pharmaceutical tablet plays a significant role in predicting the performance of the tablet when ingested. Important tablet properties, such as coating uniformity and mechanical integrity, that can be predicted based on its surface properties manifest the inevitable link between a tablet's surface property (e.g. Gloss, average surface roughness) and its efficacy. Adding to the above statements, these tablet surface properties also control, for example, the disintegration rate of the tablet. Surface roughness of a porous flat-faced tablet can be measured with a laser stylus [1]. Detection of coating thickness of a tablet is more challenging task but this issue has been

\footnotetext{
* Correspondence: prince.bawuah@uef.fi

Department of Physics and Mathematics, University of Eastern Finland, P.O. Box 111Fl-80101 Joensuu, Finland
}

nicely solved with the aid of terahertz (THz) measurement technique [2]. Photonics based tablet measurement technique has unique advantages such as the nondestructive nature and the real time detection principle. A typical pharmaceutical tablet consists of excipients and active pharmaceutical ingredient (API). There is always an interest surge by pharmacists and pharmaceutical scientists to finding ways and means of achieving inline methods/tools for monitoring the consistency in the amount of API of tablets during batch production. It is also the desire of quality monitoring and control bodies such as the Food and Drug Authority (FDA) to acquire portable and cost-effective tools for screening possible counterfeit tablets in field measurement conditions. Powerful methods that are based on near infrared (NIR) spectroscopy [3], as well as and Raman spectroscopy [4] have been adopted for the detection of API in tablet 
production environments and counterfeit drugs. These and other spectroscopic methods give information about the materials' properties of a tablet but not about the surface roughness. When a probe wave with optical wavelengths are used, one can consider surface roughness as a source of noise, which is usually manifested in cases where coherent laser radiation is utilized. An ideal pharmaceutical tablet is supposed to compose of a uniform mixture and distribution of excipients, API, and air pores across the volume of the tablet. Given such an ideal tablet, it is expected that its surface property should be a reflection of the surface distribution of these three volumetric material factors. In optical sense, this means that both the surface roughness and spatial variation of the magnitude of the refractive index over the tablet's surface contribute to the strength of incident light scattering. Hence, both the material property (e.g. refractive index) and the topographical property (e.g. surface roughness) simultaneously rule the strength and phenomena of the interaction of light with the surface of a tablet. The combined material and surface effect can be described using the concept of specular gloss, which depends on both the surface roughness and the refractive index of the tablet. From now on we use the phrase gloss to denote specular gloss. Gloss has already been utilized for the description of coated tablet [5], but as far as we know, it has not be utilized in the screening of counterfeit tablets. Detection of gloss, especially in field measurement conditions, and with minimal expertise is relatively a simple measurement method that complements data obtained by portable spectrometers, which are used for counterfeit drug detection [6].

We have developed diffractive optical-element based sensors for surface porosity inspection of two-phase pharmaceutical compacts with flat-faces [7], film coating inspection of oval shaped pharmaceutical tablets [8], single crystal inspection of a pharmaceutical compact [9], and also the detection of surface gloss (surface porosity) of a whole pharmaceutical compact with the aid of gloss map [10].

The motivation behind this paper is to study the use of two different glossmeters, which are based on the use of diffractive optical element (DOE) for the detection of surface quality of authentic and counterfeit tablets (theory, technical details and industrial applications of the DOE-based glossmeters are presented in [11-13]). For this purpose, we have studied the surface properties of commercially available antimalarial tablets. The key idea is that the surface roughness of a fake tablet differs, to a great extent, from that of an authentic tablet. In such a case one can also expect difference in the magnitude of the gloss between authentic and counterfeit tablets. For the differentiation of tablets by gloss, we have used a scanning glossmeter for a comprehensive gloss map detection, and a handheld glossmeter which is a practical instrument for both industrial environments and in field conditions for quick pointwise checking of the surface quality of both flat or curved tablets. Although the study is devoted to authentic and counterfeit tablet inspection, the DOE-based glossmeters can be used for industrial inspection of surface quality of authentic tablet products.

\section{Methods \\ Samples}

Artemether lumefantrine antimalarial tablets, including both authentic and counterfeit tablets which, according to their label, contain $20 \mathrm{mg}$ artemether and $120 \mathrm{mg}$ lumefantrine were obtained from Ghana. Four types of commercially available antimalarial tablets were used in the study. Two of these were certified authentic tablets whereas the other two types were counterfeit according to the FDA of Ghana. The counterfeit and authentic tablet samples, labeled as Fake 1 (FK1) and Genuine 1 (GN1) in this work, were manufactured by Novartis (Beijing, China) according to the labels and contain similar excipients (i.e. microcrystalline cellulose, croscarmellose sodium, magnesium stearate, polysorbate 80 and silica colloidal anhydrous). Genuine 2 (GN2) tablet samples were, according to their labels, manufactured by Novartis (Saglik, Gida ve Tarim Urunleri Sanayi ve Ticaret A.S., Istanbul Turkey) whereas Fake 2 (FK2) samples were produced by Novartis (Suffern, New York, USA) as indicated on the package. GN 2 consisted of all the excipients in GN1 in addition to hypromellose. The excipients of FK2 samples are unknown. The lack of information of excipients is already an indicator that something is wrong with this tablet.

In Table 1, we have shown basic data of the authentic and counterfeit tablets. It is obvious that there are some differences even between the properties of the authentic tablets, namely weight, height and diameter are slightly different and reflect slightly different production conditions in the two factories. Comparison of GN1 and FK1 shows rather big differences in weight and height, which are not possible regarding the variation limits of authentic tablets. Similar conclusion can be drawn also with regard to GN2 and FK2. In other words, rather simple

Table 1 Basic data of the antimalarial tablets

\begin{tabular}{lccc}
\hline Tablet samples & Weight $(\mathrm{mg})$ & Height $(\mathrm{mm})$ & Diameter $(\mathrm{mm})$ \\
\hline GN1 & 243.4 & 3.24 & 9.05 \\
GN2 & 241.4 & 3.08 & 9.09 \\
FK1 & 290.9 & 3.78 & 9.14 \\
FK2 & 255.3 & 3.25 & 9.12 \\
\hline
\end{tabular}

The weight was measured using the analytical balance Sartorius 2432 with accuracy $\pm 0.1 \mathrm{mg}$. The height and diameter were measured with Vogel digital caliber with accuracy $\pm 0.01 \mathrm{~mm}$ 
measurements can be used to screen fake antimalarial tablets from authentic ones of this study without any spectral measurements.

\section{Optical profilometer}

In this work, we have used the optical interference profilometer (WYKO NT9300), a non-contact profilometer capable of rendering fast measurements, to quantify the average surface roughness of the antimalarial tablets. Description of the principle of operation of the profilometer and how to calculate the average surface roughness $(R \mathrm{a})$ of the porous pharmaceutical compacts were presented in [10].

\section{D glossmeter}

The principle of operation of the glossmeter for scanning of a two-dimensional (2D) gloss map from a compact has been explained previously [10]. Herein, we only give a brief description of the $2 \mathrm{D}$ glossmeter. The light source is a semiconductor laser with a wavelength of $635 \mathrm{~nm}$ and an output power of $5 \mathrm{~mW}$. The choice of the laser wavelength plays a significant role on both the strength of light scattering and absorption (dispersion). However, this work utilizes inexpensive red light emitting laser due to the demand of low cost sensors especially in the developing countries. Other laser types like the green and blue lasers could have also been utilized (showing different magnitude of light scattering) on our samples. The reference for calibration of the 2D glossmeter is a commercial black glass gloss standard. The surface of each tablet was scanned three times in $x-y$ direction with a resolution of $0.05 \mathrm{~mm} /$ pixel in both directions.

In the case of the study of Ref. [10], the test samples were relatively simple, namely a training set of pharmaceutical compacts with varying porosity composing of microcrystalline cellulose (MCC) and air voids. However, there are some interesting features in that study which we wish to revisit before proceeding. One conclusion made was that the measured average surface roughness and the gloss have a linear correlation. Another observation was that although a tablet set consisted of tablets that have varied nominal porosity (volumetric property), the gloss (surface property) value was different for the opposite faces of the flat-faced compacts. In other words, such tablets have different surface roughness/surface porosity due to the compression cycle based on using a dye with upper and lower punches. Differences recorded in the surface roughness for the opposite sides of a given tablet can lead to different dissolution rate of the tablet, and also serve as the basis for screening of fake tablets as will be shown later in the this study. This is the first time we have applied the $2 \mathrm{D}$ glossmeter for the detection of gloss map and average gloss from both authentic and fake commercially available pharmaceutical tablets.

The scanning glossmeter gives information on minute gloss mottling, and hence it is possible to find out spatial differences of surface quality over the flat face of the whole tablet. Variation of the gloss map shows the presence of surface non-uniformity. This is because the obtained gloss maps were due to the reconstuction from poinwise measurement of both the surface roughness and the refractive index at the laser spot over the scanned area of the tablet surface. Such information is useful in both off-line quality control of tablets and in the screening of fake tablets. This measurement method can also be applied in the case of curved tablets, but then a small part of the tablet surface is subjected to inspection. Using another type of setup, which we suggested for forensic studies [14], it is not impossible to get gloss map from oval shaped tablets. However, this measurement is out of scope of the present samples that are flat-faced except the presence of a groove and other patterns on the faces of the tablets.

\section{Handheld glossmeter}

In case of achieving a fast gloss inspection of pharmaceutical tablets, the scanning glossmeter fails since it is timeconsuming and may fail to deliver correct results when exploited in field measurement conditions of counterfeit tablets. In order to achieve fast measurement requirements, we have developed a handheld glossmeter, which is based on the same measurement principle as the scanning glossmeter. In addition, the handheld glossmeter can be used for detecting gloss from curved objects, such as curved metal objects [15] (there also exist the theory of gloss of curved object). This study is the first of its kind where we have applied the handheld glossmeter for the detection of gloss from pharmaceutical tablets. In this study, we have measured gloss from five different locations on both faces of authentic and fake antimalaria tablets. The use of the handheld glossmeter is illustrated in Fig. 1. The same gloss standard as mentioned above is used for the calibration of the handheld glossmeter, and similar semiconductor laser serves as the light source. The accuracy of the reading is \pm 0.1 for gloss values less than 100 .

We tested the validity of the handheld glossmeter for the surface quality inspection of commercial pharmaceutical tablets, i.e., authentic and fake antimalarial tablets, because the portable rechargeable battery operated handheld gloss meter can serve as a tool for field testing of tablets due to its ease of operation that requires no experts as compared to the use of spectroscopic devices.

\section{Results and discussion}

Figure 2 shows a false color image of the surface topography of authentic and counterfeit antimalarial tablet 


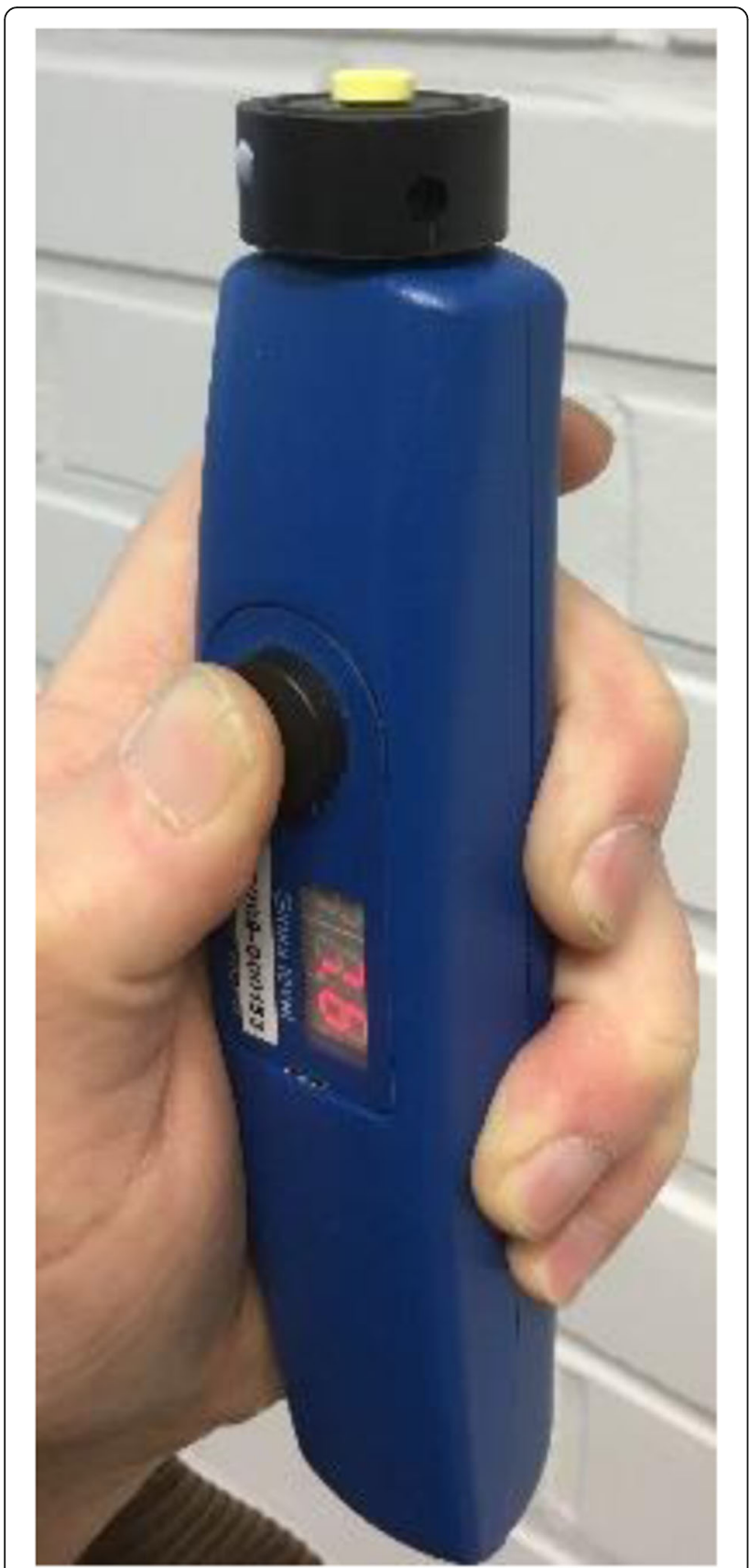

Fig. 1 A photo of the handheld glossmeter depicting how the measurement on the tablet samples were taken. The thumb is over the measurement button and the gloss reading is shown on the display

samples measured using the optical interference profilometer. From the image data of Fig. 2, and by visual inspection, it is possible to find regions with high or low reflectivity. In Table 2 , the values of the average surface roughness $\left(R_{a}\right)$ of the present samples are given. It is evident that the average surface roughness of same product but from two different factories shows differences as measured from sides $\mathrm{A}$ and $\mathrm{B}$. However, the $R_{a}$ is much lower with the authentic tablets than with the fakes. This can be understood by the fact that the authentic tablets have been compressed with more sophisticated facilities than the fake ones. One important factor is the high compression force used in the compression of authentic tablets which is the reason behind the relatively low surface roughness measured from both sides of the genuine tablets. Another probable reason can be attributed to the poor surface quality of the punches used for the compression of fake tablets. The high surface roughness of FK1 and FK2 tablets is obvious from the data of Table 2, and such high values can be used for screening of the fake from the genuine tablets. Anyhow, a common feature with both the authentic and the fake tablets is that the surface roughness of the A side is lower than the B side. This is due to the compression cycle of the tablet.

In Fig. 3, the gloss maps of the surface of tablet samples are shown. With the resolution of $0.05 \mathrm{~mm} /$ pixel, it took about 3 mins to scan an area of $12.5 \mathrm{~mm} \times$ $17.5 \mathrm{~mm}$ along the $x-y$ direction as shown in Fig. 3. It is worth noticing that the gloss maps of Fig. 3 captures extra surroundings around the tablets. However, it is interesting and promising to mention that, it took less than a minute (c.a. $53 \mathrm{~s}$ ) to scan an area of $6 \mathrm{~mm} \times$ $6 \mathrm{~mm}$ considering the tablets' diameter of $9 \mathrm{~mm}$. The values of the gloss (see Table 2) for various samples were obtained by selecting three scanned areas from the gloss map and afterwards averaged to give the final gloss value of a given sample. From the gloss maps of Fig. 3, it is obvious that the symbol "CO" on side A, and the groove on side $\mathrm{B}$ in genuine tablets can be distinguished. However, in the case of the counterfeit tablets these mark are not so visible. This image information can be used for screening of fake antimalarial tablets. Moreover, the calculated average gloss is higher with the authentic than the counterfeit tablets. The surface roughness of GN2 on both sides is lower than with GN1. Therefore, one would expect that the average gloss of GN2 would be higher than with GN1, but this is not the case. As we have already mentioned gloss depends on both surface roughness and the spatial refractive index. In GN2 there is hypermellose which is absent from GN1. This can be a reason, or alternatively different surface distribution of excipients and the API between the two authentic tablets. Anyhow this is also an interesting feature that can be detected by the measurement of gloss. By comparing the average gloss of GN1 and FK1 as well as GN2 and FK2, one observes relatively big differences between them. From the above consistency observed between the surface roughness data and that of the gloss for the tablet samples used in this study, one can obviously infer that the $2 \mathrm{D}$ gloss reading can be used in conjunction 

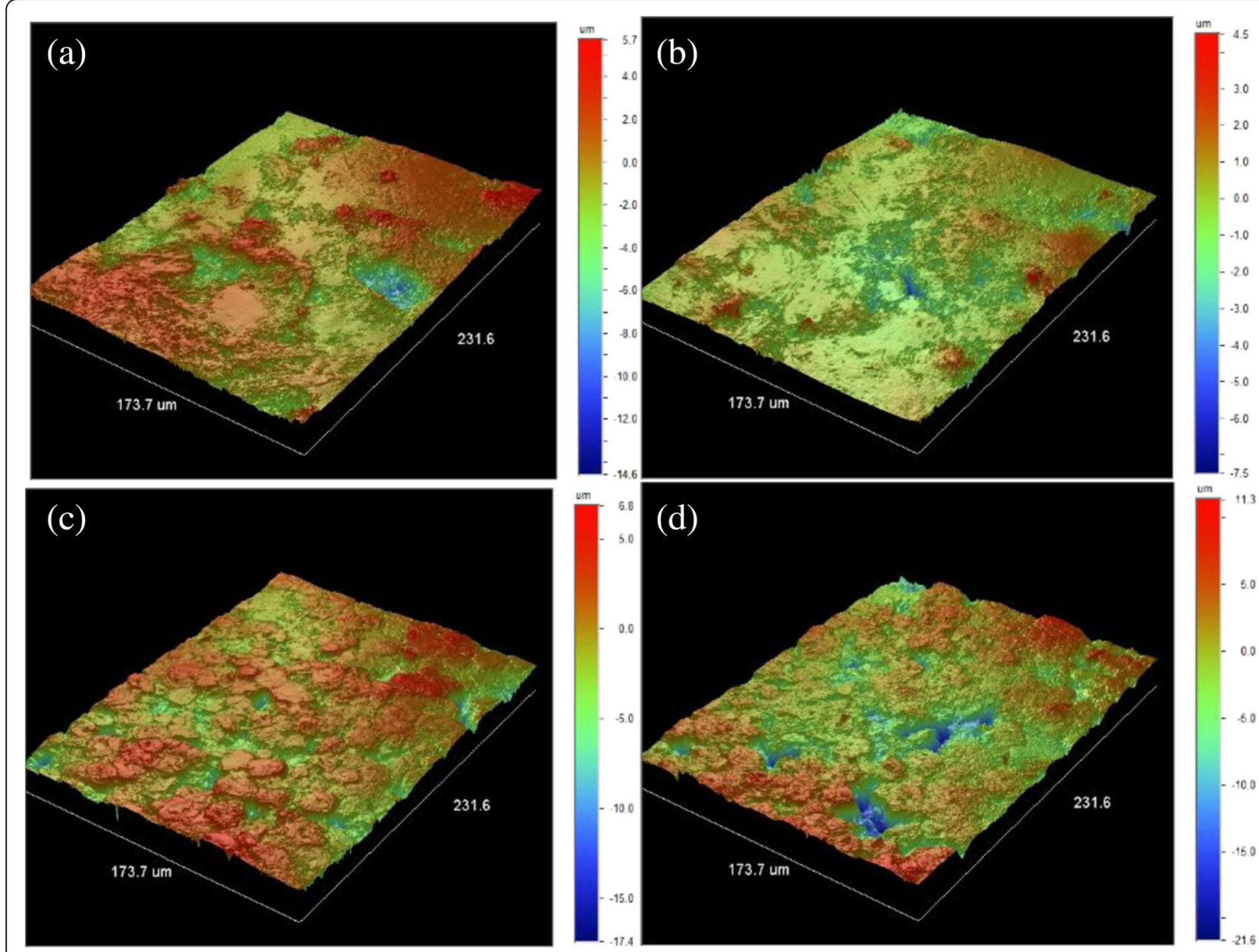

Fig. 2 Surface roughness maps of side A of the tablet samples. $\mathbf{a}$ and $\mathbf{b}$ represent the Genuine 1 and 2 whereas (c) and (d) are the Fake 1 and 2 respectively

with the average surface roughness, to give more evidence when screening fake tablets. In the case of FK2 the average surface roughness of sides A and B are close to each other as well as the $2 \mathrm{D}$ gloss, but the gloss data suggests that there is some excipient on side B which may explain slightly higher gloss with $B$ than $A$.

The gloss (i.e. the handheld gloss, HHGloss) was measured from five different locations of each tablet using the handheld glossmeter. The data of Table 2 for HHGloss was obtained by averaging five measurements, following the scheme used in the detection of the average surface roughness, e.g., in metal industry with the aid of a handheld profilometer. The gloss readings of 2D and handheld glossmeters are different due to different optical geometry of the glossmeters. Data of the handheld device is consistent with the data measured with the 2D glossmeter, and also with the optical profilometer. This is encouraging, namely a quality inspector can make quick off-line detection (which is typical method in the factories) of the quality of a tablet (the tablet makers define decision limits for gloss). Furthermore, the various observations made in this study is quite promising since quality and control agencies can fast track and screen counterfeit and substandard pharmaceutical tablets

Table 2 Average surface roughness $\left(R_{a}\right)$, average gloss (2D gloss) and average of gloss detected by the handheld glossmeter

\begin{tabular}{lcccccc}
\hline Tablet samples & Ra (side A) & Ra (side B) & 2D gloss (Side A) & 2D gloss (Side B) & HHGloss (Side A) & HHGloss (Side B) \\
\hline GN1 & 0.981 & 1.375 & 0.4068 & 0.3712 & 8.5 & 7.7 \\
GN2 & 0.699 & 0.791 & 0.3799 & 0.3039 & 7.9 & 7.1 \\
FK1 & 1.871 & 2.964 & 0.3161 & 0.2934 & 7.7 & 7.0 \\
FK2 & 2.235 & 2.368 & 0.2936 & 0.3026 & 6.9 & 6.9 \\
\hline
\end{tabular}



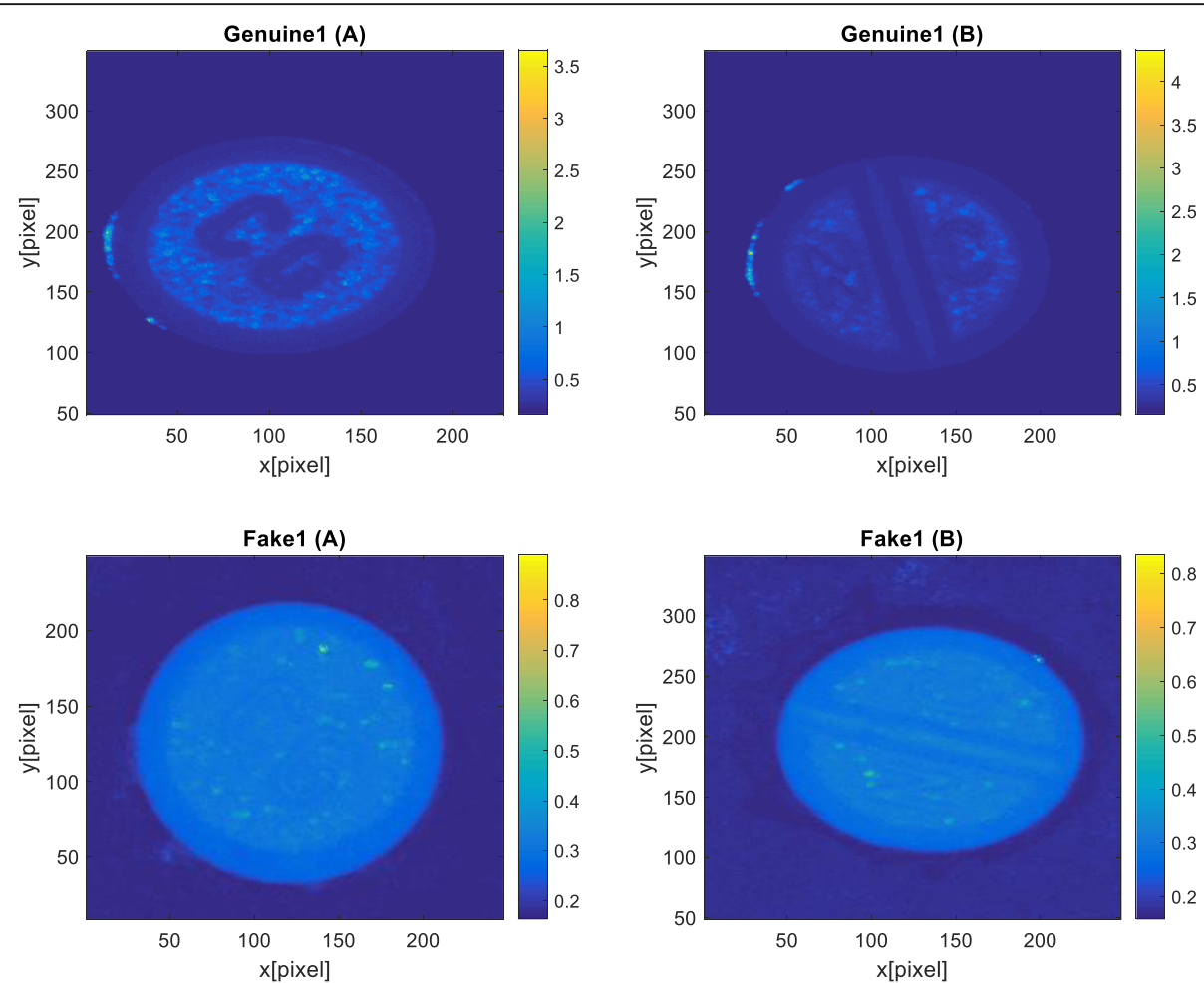

Fig. 3 The 2D gloss maps of authentic and counterfeit antimalaria tablet samples measured from A and B sides. The color bar shows the magnitude of the gloss

flooding the markets of developing counties just by using this simple and inexpensive device.

\section{Conclusions}

We first measured weight and dimensions of the tablets, which already indicated the presence of fakes. The measurement of average surface roughness gave more confidence that the fake tablets have poorer surface quality than the authentic tablets. Pharmaceutical tablets are rather complicated due to the porosity and presence of several excipients and the API. This fact can be exploited in optical measurement of gloss which is a convolution of surface roughness and the refractive index of the tablet. Both surface roughness and refractive index may vary spatially. In case of an authentic tablet usually the surface quality is better than with the fake tablet. This became evident by the measurement of gloss from tablets with two different glossmeters, namely a scanning glossmeter and a handheld model. We propose that the 2D glossmeter is a useful tool for pharmacist regarding both research and practical off-line quality inspection of pharmaceutical tablets, whereas the handheld glossmeter can be useful for quick inspection of randomly chosen tablets from the production line, and also for screening of counterfeit tablets. The problem of counterfeit tablets is not only a problem in developing but also in developed countries.
Abbreviations

2D: Two-dimension; API: Active pharmaceutical ingredient; DOE: Diffractive optical element; FDA: Food and Drug Authority; HHGloss: Handheld gloss; MCC: Microcrystalline cellulose; NIR: Near infrared; Ra: Average surface roughness; THz: Terahertz

\section{Acknowledgement}

We thank Mr. Issaka Nii Amu Collison-Cofie, Regulatory Officer at Food and Drugs Authority (FDA) Ghana, for the samples provided for this work.

\section{Funding}

Not applicable.

\section{Availability of data and materials}

Detail about data has been provided in the manuscript.

\section{Authors' contributions}

The measurement and analysis of the 2D gloss, the handheld gloss and the surface roughness data were done by PB (PhD), KP (Prof.) and PP (PhD) respectively. The writing of the manuscript was done by PB and K-EP. All authors read and approved the final manuscript.

\section{Competing interests}

The authors declare that they have no competing interests.

\section{Consent for publication}

Not applicable.

Ethics approval and consent to participate Not applicable.

\section{Publisher's Note}

Springer Nature remains neutral with regard to jurisdictional claims in published maps and institutional affiliations. 
Received: 21 March 2017 Accepted: 6 June 2017

Published online: 15 June 2017

\section{References}

1. Healy, A.M., Corrigan, O.I., Allan, J.E.M.: The effect of dissolution on the surface texture of model solid-dosage forms as assessed by non-contact laser profilometry. Pharm Technol Eur 9, 14-22 (1995)

2. May, R.K., Evans, M.J., Zhong, S., Warr, I., Gladden, L.F., Shen, V., Zeitler, J.A.: Terahertz in-line sensor for direct coating thickness of individual tablets during film coating in real-time. J Pharm Sci 100, 1535-1544 (2011)

3. Storme-Paris, I., Rebiere, H., Matoga, M., Civade, C., Bonnet, P.-A., Tissimer, M. $H_{\text {., }}$ Chaminade, P.: Challenging near infrared spectroscopy discriminating ability of counterfeit pharmaceutical detection. Anal Chim Acta 658, 163173 (2010)

4. Roggo, Y., Dgardin, K., Margot, P.: Identification of pharmaceutical tablets by Raman spectroscopy and chemometrics. Talanta 81, 988-995 (2010)

5. Rowe, R.C.: Gloss measurement on film coated tablets. J Pharm Pharmacol 37, 761-765 (1985)

6. Martino, R., Malet-Martino, M., Gilard, V., Balayssac, S.: Counterfeit drugs: analytical techniques for their identification. Anal Bioanal Chem 398, 77-98 (2010)

7. Silvennoinen, R., Peiponen, K.-E., Laakkonen, P., Ketolainen, J., Suihko, E., Paronen, P., Räsänen, J., Matsuda, K.: On the optical inspection of the surface quality of pharmaceutical tablets. Meas Sci Technol 8, 550-554 (1997)

8. Peiponen, K.-E., Silvennoinen, R., Räsänen, J., Matsuda, K., Tanninen, V.P.: Optical coating inspection of pharmaceutical tablets by diffractive element. Meas Sci Technol 8, 815-818 (1997)

9. Juuti, M., van Veen, B., Peiponen, K.-E., Ketolainen, J., Kalima, V., Silvennoinen, R., Pakkanen, T.: Local and average gloss from flat-faced sodium chloride tablets. AAPS PharmSciTech 7, E1-E6 (2006)

10. Bawuah, P., Pierotic Mendia, A., Silfsten, P., Pääkkönen, P., Ervasti, T., Ketolainen, J., Zeitler, J.A., Peiponen, K.-E.: Detection of porosity of pharmaceutical compacts by terahertz radiation transmission and light reflection measurement techniques. Int J Pharm 465, 70-74 (2014)

11. Silvennoinen, R., Peiponen, K.-E., Myller, K.: Specular Gloss. Elsevier, Oxford (2008)

12. Kuivalainen, K, Oksman, A, Juuti, M., Myller, K, Peiponen, K-E: Advanced glossmeters for industrial applications. Opt Rev 17, 248-251 (2010)

13. Oksman, A., Kuivalainen, K., Tåg, C.-M., Juuti, M., Mattila, R., Hietala, E., Gane, P.A.C., Peiponen, K.-E.: Diffractive optical element-based glossmeter for the on-line measurement of normal reflectance of printed porous coated paper. Opt Eng 50, 043606 (2011)

14. Kuivalainen, K., Peiponen, K.-E., Myller, K.: Application of a diffractive element-based sensor for detection of latent fingerprints from a curved smooth surface. Meas Sci Technol 20, 077002 (2009)

15. Kuivalainen, K., Oksman, A., Peiponen, K.-E.: Definition and measurement of statistical gloss parameters from curved objects. Appl Opt 49, 5081-5086 (2010)

\section{Submit your manuscript to a SpringerOpen ${ }^{\circ}$ journal and benefit from:}

- Convenient online submission

- Rigorous peer review

- Open access: articles freely available online

- High visibility within the field

- Retaining the copyright to your article 